\title{
AN IMPROVED BEARING ESTIMATION ALGORITHM FOR RANDOMLY PERTURBED ARRAYS WITHOUT CALIBRATION
}

*Yih-Min Chen, Ju-Hong Lee, and Chien-Chung Yeh

Department of Electrical Engineering

National Taiwan University

Taipei, Taiwan, Republic of China

Abstract

The performance of high-resolution bearing estimation algorithms is greatly sensitive to the presence of sensor position errors. In [1], calibration-free algorithms, which perform bearing estimation based on a Toeplitz covariance matrix reconstructed from the observed one, were proposed for a linearly periodic array whose the sensor locations are randomly perturbed. In this paper, we improve the methods of [1] by utilizing an iterative algorithm to reconstruct a covariance matrix satisfying both Toeplitz and eigenstructure properties from the observed one. Computer simulations show that the iterative method is superior to the method in [1] at the price of computations.

I. Introduction

High-resolution bearing estimation techniques are usually developed under the assumption that the locations of array sensors are known precisely. When there are uncertainties in sensor location, the performance of these techniques tends to deteriorate greatly due to the inconsistency between the true and assumed sensor locations. Conventionally, this problem is tackled by calibrating the sensor locations prior to performing bearing estimation. However, the proposed calibration procedures require additional computations and must fulfill certain limitations, such as the number of deployed sources and the geometry of array. Hence it is important to develop an algorithm which does not need the calibration procedure.

In [1], a calibration-free algorithm for a nominally linearly periodic array with randomly perturbed sensor locations was proposed. The approach reconstructs a covariance matrix from the observed one and then performs bearing estimation based on this reconstructed covariance matrix. Since the ideal covariance matrix of the nominal array is Toeplitz, the methods of [1] use the TAM [2] and its modification (MTAM) to implement the reconstruction procedure. In this paper, we further extend this approach by taking into account the property of the ideal covariance matrix, i.e. the reconstructed covariance matrix has not only Toeplitz form but also a multiplicity of $L-M$ for its minimum eigenvalue, where $L$ and $M$ are the numbers of sensors and sources, respectively. An iterative algorithm is proposed to implement this covariance matrix reconstruction procedure. Simulation results show that the performance is improved. 
II. A Review of the TAM Method

For a linearly periodic array, the id eal covariance matrix is given as

$$
\begin{aligned}
& \mathrm{R}_{I}=\left[\mathrm{r}_{1 i j}\right] \\
& \mathrm{r}_{1 \mathrm{ij}}=\sum_{\mathrm{m}=1}^{\mathrm{M}} \mathrm{E}\left[\left|\alpha_{\mathrm{m}}(\mathrm{t})\right|^{2}\right] \mathrm{e}^{\mathrm{j} \mathrm{k}_{\mathrm{c}}(\mathrm{i}-\mathrm{j}) \mathrm{d} \sin \theta_{\mathrm{m}}+\sigma^{2} \delta_{\mathrm{ij}},}
\end{aligned}
$$

where $\alpha_{\mathrm{m}}(\mathrm{t})$ and $\theta_{\mathrm{m}}$ are the $\mathrm{mth}$ signal amplitude and arrival angle relative to array broadside, respectively, $\mathrm{d}$ is the interelement spacing, $\mathrm{k}_{\mathrm{c}}$ the wavenumber, and $\sigma^{2}$ is the power of noise. However, when there exists random perturbation, the observed covariance matrix is given as

$$
\begin{aligned}
R= & \left\{r_{i j}\right] \\
r_{i j}= & \sum_{m=1}^{M} E\left[\left.l \alpha_{m}(t)\right|^{2}\right] \mathrm{e}^{j k_{c}(i-j) d \sin \theta_{m}} \\
& \cdot e^{j k_{c}\left(\Delta \underline{u}_{i}-\Delta \underline{u}_{j}\right)^{t} \underline{\theta}_{m}+\sigma^{2} \delta_{i j}}
\end{aligned}
$$

where $\Delta \underline{u}_{i}=\left[\Delta \mathrm{x}_{\mathrm{i}}, \Delta \mathrm{y}_{\mathrm{i}}\right]^{\mathrm{t}}$ and $\underline{\theta}_{\mathrm{m}}=\left[\sin \theta_{\mathrm{m}}, \cos \theta_{\mathrm{m}}\right]^{\mathrm{t}}$ are the position errors of the ith sensor and the bearing vector of the mth source, respectively. In [1], it has been shown that Toeplitzizing the observed covariance matrix, i.e.

$$
\begin{aligned}
& \hat{\mathrm{R}}_{\mathrm{T}}=\mathrm{T}[\mathrm{R}]=\left[\hat{\mathrm{r}}_{\mathrm{Tij}}\right] \\
& \hat{\mathrm{r}}_{\mathrm{T} \mathrm{ij}}=\hat{\mathrm{r}}_{\mathrm{T}}(\mathrm{i}-\mathrm{j})
\end{aligned}
$$

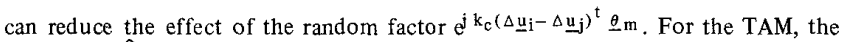
elements of $\hat{\mathrm{R}}_{\mathrm{T}}$ are given as

$$
\hat{\mathrm{r}}_{\mathrm{T}}(-\mathrm{n})=\frac{1}{\mathrm{~L}-\mathrm{n}} \sum_{\ell=1}^{\mathrm{L}-\mathrm{n}} \mathrm{r}_{\ell(\ell+\mathrm{n})}, \quad 0 \leqslant \mathrm{n}<\mathrm{L}
$$

while for the MTAM, the elements of $\hat{\mathrm{R}}_{\mathrm{T}}$ are replaced by

$$
\begin{aligned}
& \hat{\mathrm{r}}_{M T}(-n)=\beta(-n) \mathrm{e}^{j \alpha(-n)} \\
& \text { where } \quad \beta(-n)=\frac{1}{\mathrm{~L}-\mathrm{n}} \sum_{\ell=1}^{\mathrm{L}-\mathrm{n}}\left|r_{\ell(\ell+n)}\right| \text { and } \alpha(-n) \triangleq \operatorname{ARG}\left(\hat{\mathrm{r}}_{T}(-n)\right) .
\end{aligned}
$$

\section{The Iterative Algorithm}

As noted in the previous section, the reconstructed covariance matrix satisfies only the Toeplitz property but not eigenstructure property in general. Therefore, the capability of the TAM can be improved if we could reconstruct a covariance matrix satisfying both properties. Since it is difficult to reconstruct such a covariance matrix directly from the observed one, we proposed an iterative algorithm, which reconstructs the Toeplitz property and eigenstructure property 
alternatively, to implement the reconstruction procedure. When the convergence has been reached, a covariance matrix satisfying both properties can be obtained. The iterative algorithm is summarized below.

a) Estimate $R$ from the received signal, let $i=0$ and $\hat{R}_{E}^{(0)}=R$.

b) Apply the TAM (or MTAM) to obtain $\widehat{R}_{T}^{(i+1)}$ from $\hat{R}_{E}^{(i)}$, i.e. $\hat{R}_{T}^{(i+1)}=T\left[\hat{R}_{E}^{(i)}\right]$.

c) Apply the eigen-reconstruction operation to get $\hat{R}_{\mathrm{E}}^{(\mathrm{i}+1)}=\bar{E}\left[\hat{\mathrm{R}}_{\mathrm{T}}^{(\mathrm{i}+1)}\right]$.

d) If $\left\|\hat{R}_{E}^{(i+1)}-\hat{R}_{T}^{(i+1)}\right\|^{2}>\epsilon$, then let $\mathrm{i}=\mathrm{i}+1$ and repeat $\left.\mathrm{b}\right)-\mathrm{d}$ ).

e) Let $\widehat{\mathrm{R}}_{\mathrm{IT}}=\widehat{\mathrm{R}}_{\mathrm{E}}^{(\mathrm{i}+1)}$ or $\hat{\mathrm{R}}_{\mathrm{T}}^{(\mathrm{i}+1)}$.

Where $\mathrm{T}[\mathrm{]}$ denotes the Toeplitzization operation defined by (5) - (7) or (5) (8), and $\overline{\mathrm{E}}[\mathrm{]}$ denotes the eigen-reconstruction operation defined as

$$
\hat{\mathrm{R}}_{\mathrm{E}}=\overline{\mathrm{E}}[\mathrm{R}]=\sum_{\mathrm{m}=1}^{\mathrm{M}} \lambda_{\mathrm{m}} \underline{\mathrm{q}}_{\mathrm{m}} \underline{\mathrm{q}}_{\mathrm{m}}^{+}+\lambda_{\mathrm{av}} \sum_{\ell=\mathrm{M}+1}^{\mathrm{L}} \underline{\mathrm{q}}_{\ell} \underline{\mathrm{q}}_{\ell}^{+}
$$

where $\lambda_{1} \geqslant \lambda_{2} \geqslant \ldots . \geqslant \lambda_{L}$ and $\underline{q}_{\ell}, \ell=1,2, \ldots, L$ are the eigenvalues and the corresponding eigenvectors of $R$, respectively, and $\lambda_{a v}$ is given as

$$
\lambda_{\mathrm{av}}=\frac{1}{\mathrm{~L}-\mathrm{M}} \stackrel{\sum}{\mathrm{L}} \mathrm{M}+1_{\ell} \lambda_{\ell}
$$

The convergence of this iterative algorithm (ITAM or IMTAM) has been shown in [3].

IV. Simulation Results

A linearly periodic array with $L=20$ and $d=\lambda / 2(\lambda=$ wavelength $)$ was used. The random perturbations $\left\{\Delta \mathrm{x}_{\ell}, \Delta \mathrm{y}_{\ell}\right\}, \ell=1,2, \ldots, \mathrm{L}$ were generated from a Gaussion random number generator with $\sigma_{\mathrm{e}}^{2}=0.02 \lambda^{2} .100$ snapshots were taken to estimate the covariance matrix $R$ in each example. Table $I$ shows the number of failures for each method in resolving directions of arrival (DOA) based on 500 independent simulations with two sources separated $10^{\circ}$ and $5^{\circ}$ in bearing, respectively. All sources are incoherent and equally powered with $\mathrm{SNR}=10 \mathrm{~dB}$. Here a method is said to "fail to resolve" the DOA if each location of the two largest peaks in the angular spectrum is not within $\pm 2.5^{\circ}$ from its true location. Figures 1 and 2 show the resulting angular spectra of a certain simulation in each example using the MUSIC, TAM, MTAM, ITAM, and IMTAM. It is noted that the performance is improved by using the iterative algorithm.

References:

[1] Y.M. Chen, J.H. Lee, and C.C. Yeh, "Calibration-free bearing estimation for arrays with randomly perturbed sensor locations," Proc. ICASSP, New York, U.S.A., pp. 2917-2920, April 1988.

[2] S.Y. Kung, C.K. Lo, and R. Foka, "A Toeplitz Approximation Approach to Coherent Source Direction Finding," Proc. ICASSP, Tokyo, Japan, pp. 193196, April 1986.

[3] Y.M. Chen, J.H. Lee, and C.C. Yeh, "Bearing estimation without calibration for randomly perturbed arrays," submitted to IEEE Trans. on ASSP. 
TABLE 1. Monte Carlo Simulation Results for

Resolving the DOA's of Two Sources.

No. of Failures in 500 Independent Trials.

Case 1: $10^{\circ}$ Separation. Case 2: $5^{\circ}$ Separation.

\begin{tabular}{|c|c|c|c|c|}
\hline The Method & TAM & MTAM & ITAM & IMTAM \\
\hline Case 1 & 42 & 15 & 10 & 5 \\
\hline Case 2 & 86 & 27 & 20 & 11 \\
\hline
\end{tabular}

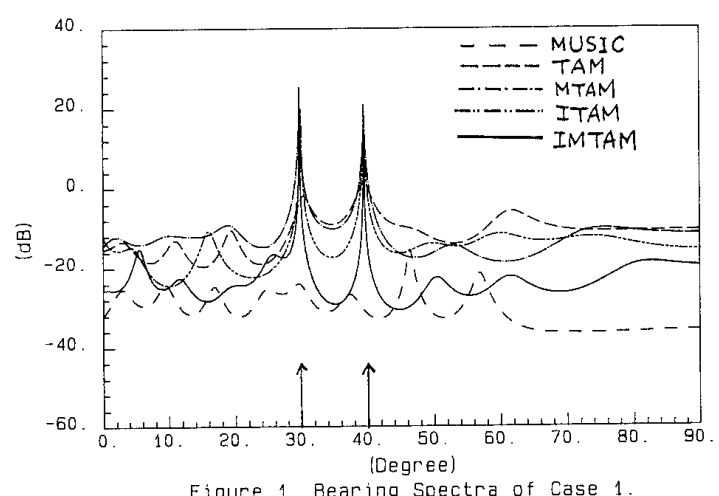

Figure 1. Bearing Spectra of Case 1.

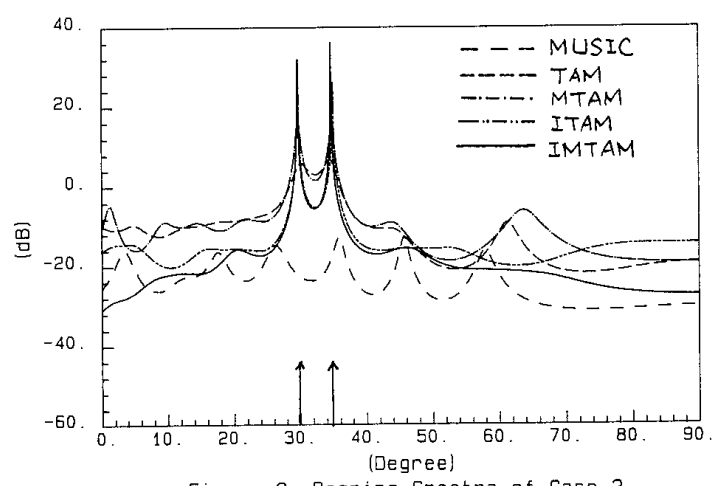

Figure 2. Bearing Spectra of Case 2. 